\title{
Rank-Based Ant System via the Relative Position in a Local Hierarchy
}

\author{
Tomoko Sakiyama $\mathbb{D}^{1},{ }^{1}$ Kotaro Uneme, ${ }^{2}$ and Ikuo Arizono $\mathbb{D}^{2}$ \\ ${ }^{1}$ Department of Information Systems Science, Faculty of Science and Engineering, Soka University, Tokyo 192-8577, Japan \\ ${ }^{2}$ Graduate School of Natural Science and Technology, Okayama University, Okayama 700-8530, Japan \\ Correspondence should be addressed to Tomoko Sakiyama; tmk.sakiyama@gmail.com
}

Received 21 June 2021; Accepted 30 August 2021; Published 8 September 2021

Academic Editor: Yu-Wang Chen

Copyright (c) 2021 Tomoko Sakiyama et al. This is an open access article distributed under the Creative Commons Attribution License, which permits unrestricted use, distribution, and reproduction in any medium, provided the original work is properly cited.

\begin{abstract}
$A S_{\text {rank }}$ has been proposed as an improved version of the ant colony optimisation (ACO) model. However, $A S_{\text {rank }}$ includes behaviours that do not exist in the actual biological system and fall into a local solution. To address this issue, we developed $A S_{\text {multi }}$ a new type of $A S_{\text {rank, }}$, in which each agent contributes to pheromone depositions by estimating its rank by interacting with the encountered agents. In this paper, we attempt further improvements in the performance of $A S_{\text {multi }}$ by allowing agents to consider their position in a local hierarchy. Agents in the proposed model $\left(A S_{\text {hierarchy }}\right)$ contribute to pheromone depositions by estimating the consistency between a local hierarchy and global (system) hierarchy. We show that, by using several TSP datasets, the proposed model can find a better solution than $A S_{\text {multi }}$.
\end{abstract}

\section{Introduction}

Ant colony optimisation (ACO) is a well-known strategy for determining short tours in the travelling salesman problem (TSP) [1]. Many ACO models have been developed by extending the ant system $(A S)$ proposed by Dorigo [2-6]. Rank-based ant system $\left(A S_{\text {rank }}\right)$ is one of the representative models that extend the original $A S$ [7]. Using the pheromone procedure update for exploring simulations, this model finds a solution to the TSP. In $A S_{\text {rank, }}$, ant agents are ranked in the order of shorter tour lengths at the end of each tour. Only the top-ranking agents are allowed to deposit pheromones. This function improves the convergence of the system by adding pheromones on only the edges toured by top-ranked agents. Thus, the system converges faster by concentrating pheromones on specific edges. However, because pheromones tend to concentrate solely on specific edges, the system frequently falls into local solutions. Furthermore, while $A S_{\text {rank }}$ is inspired by the behaviour of real ants, the viewpoint of a third party is introduced outside the system to classify agents in a single hierarchy, i.e., to place agents in the order of tour lengths. Agents that can complete tours in less time are rewarded with higher positions in the hierarchy.

To eliminate the viewpoint of the third party from the system, we recently proposed a new type of $A S_{\text {rank }}$ named $A S_{\text {multi }}$ in which an individual agent constructs a subjective hierarchy based on the interaction with encounters [8]. In $A S_{\text {multi }}$, agents decide whether to deposit pheromones based on a unique hierarchy for each agent, i.e., deposit pheromones when they encounter other agents that have worse solutions than theirs. Furthermore, if two agents meet one other during a tour, a subjective/unique hierarchy is used to rank them and other agents according to the tour lengths. To this end, individuals have a unique list of ranked agents based not on the viewpoint of the third party but on the firstperson view. We found that such a contrivance allows the $A S_{\text {multi }}$ system to find better solutions than $A S_{\text {rank }}$. However, agents in $A S_{\text {multi }}$ sometimes fall into a local solution caused by the mismatch between their subjective hierarchies and the system hierarchy, i.e., the actual single rank of the agents' tours. Therefore, there is room for further improvement in $A S_{\text {multi }}$. 
In this study, we propose an improved version of $A S_{\text {multi }}$. We name this as $A S_{\text {hierarchy. }} A S_{\text {hierarchy }}$ follows $A S_{\text {multit; }}$; however, agents in $A S_{\text {hierarchy }}$ sometimes deposit pheromones even when they encounter other agents with better solutions than themselves. This occurs because agents consider the mismatch between their local hierarchies and the global (system) hierarchy as individual agents cannot know the absolute rank in the system hierarchy. From the viewpoint of a specific agent, the greater the number of agents offering better solutions than the agent who encounters them, the fewer the opportunities for the agent to get away from a local solution. Therefore, the agents are allowed to deposit pheromones to some extent in cases where they encounter other agents having better solutions. Thus, individual agents in living systems seem to adapt as they consider the local information [9]. Through numerical simulations using several TSP datasets, we show that the proposed model outperforms $A S_{\text {multi. }}$

Eliminating the viewpoint of the third party from the system is related to the subpopulations. Several studies have employed this approach for some optimisation problems [10-13]. For example, Cai et al. used the cooperative coevolution strategy to improve the global search capability for airport gate allocation optimisation [10]. Deng et al. used a similar mechanism, i.e., the CC framework, to deal with large-scale problems [11]. Multipopulations or population diversity contribute toward finding the global optimal solution. In our method, individual agents independently modulate their pheromone depositions based on their experiences during each tour.

\section{Materials and Methods}

2.1. Multirank-Based $A S\left(A S_{\text {mult }}\right)$. We propose a multirankbased ant system $\left(A S_{\text {multi }}\right)$ [8] as an improved version of $A S_{\text {rank }}$ [7]. In $A S_{\text {multi }}$, the probability of the next destination selection determined in the same manner as in $A S_{\text {rank }}$, but a new approach is used for regulating agents who contribute to pheromone deposition. The new method of pheromone update in $A S_{\text {multi }}$ is explained here. First, we define the parameters as follows:

$s$ : step count in one tour

$\theta_{\text {step }}$ : threshold for tour step in agent competition

ratio: the value calculated in agent competition

$\varphi_{\text {ratio }}$ : threshold for ratio in agent competition

$C_{k}$ : the binary parameter indicating whether agent $k$ survived the competition

$N_{\text {agent }}$ : the number of agents (equal to $N_{\text {city: }}$ the number of cities)

$N_{\text {city: }}$ the set of cities

$\operatorname{city}_{x}(s, t)$ : city where the agent $x$ is in at step $s$

$\operatorname{city}_{y}(s, t)$ : city where the agent $y$ is in at step $s$

$\ell_{x}(s, t)$ : accumulated tour length of agent $x$ at step $s$

$\ell_{y}(s, t)$ : accumulated tour length of agent $y$ at step $s$

$L_{k}$ : tour length of the solution found by agent $k$
$T_{k}$ : set of edges included in the solution found by agent $k$

If the following two conditions are satisfied, then a competition occurs among agents:

(i) The parameters satisfy the relationship $s / N_{\text {city }}>\theta_{\text {step }}$

(ii) There are other agents in the same city

When one agent $x$ encounters another agent $y$, i.e., city $(s, t)=\operatorname{city}_{y}(s, t)$, the agents mutually compare the accumulated tour lengths at tour step $s$ of this tour to determine whether there is enough difference between their accumulated tour lengths. Using their accumulated tour lengths $\ell_{x}$ $(s, t)$ and $\ell_{y}(s, t)$, an evaluation value ratio is calculated. If the ratio exceeds the threshold in agent competition $\varphi_{\text {ratio }}$ (ratio $>\varphi_{\text {ratio }}$ ), one long-length solution is defeated. In this case, 0 is assigned to the parameter $C_{k}$ of the defeated agent. Here, the competition process is defined as follows:

$$
\begin{gathered}
\text { ratio }=\frac{\left|l_{x}(s, t)-l_{y}(s, t)\right|}{l_{x}(s, t)+l_{y}(s, t)}, \\
C_{x} \leftarrow 0, \quad \text { if ratio } \leq \varphi_{\text {ratio }}, \ell_{x}(s, t) \geq \ell_{y}(s, t), \\
C_{y} \leftarrow 0, \quad \text { if ratio } \leq \varphi_{\text {ratio }}, \ell_{x}(s, t) \leq \ell_{y}(s, t) .
\end{gathered}
$$

The following prerequisites should be noted as well:

(i) All parameters of $C_{k}$ are initialised to 1 at the beginning of a tour

(ii) If an agent $x$ is defeated even once in the middle of a tour, $C_{x}$ remains 0 until the end of that tour

(iii) If three or more agents exist simultaneously in the same city, comparison for agent $k$ is performed in all pairs until $C_{k}$ is replaced with 0

Finally, some agents who have never been defeated after completing a tour $\left(C_{k}=1\right)$ can perform pheromone deposition. In this method, each agent decides about whether to take part in pheromone deposition using the information from contacted agents. As this method does not require the viewpoint of the external third party for summarising the agent's information, such as $A S_{\text {rank }}$, it is faithful to the actual habits of ant swarms. After creating a tour, the pheromone update process is executed as follows:

$$
\tau_{i j}(t+1)=\rho \tau_{i j}(t)+ \begin{cases}\sum_{k} \frac{1}{L_{k}}, & \text { if }(i, j) \in T_{k}(t), C_{k}=1, \\ 0, & \text { otherwise. }\end{cases}
$$

Agents in $A S_{\text {multi }}$ cannot perform pheromone deposition when they encounter other agents with better information. To this end, agents determine whether they are in a good/bad position in the global (system) hierarchy by using the subjective local hierarchy to confirm whether they have better/worse information than the agents encountered. However, identifying the local hierarchy as a subhierarchy of 
the global hierarchy does not enable convergence to specific solutions. To solve this problem, we proposed $A S_{\text {hierarchy. }}$.

2.2. Hierarchical Rank-Based AS (AS hierarchy $_{\text {) }}$. Here, we describe the hierarchical rank-based ant system $\left(A S_{\text {hierarchy }}\right)$ proposed in this study. Agents in $A S_{\text {hierarchy sometimes }}$ deposit pheromones even if they have worse solutions. From the viewpoint of a specific agent, the greater the number of encountered agents offering better solutions, the fewer the opportunities to get away from a local solution. To this end, agents in the proposed $A S_{\text {hierarchy }}$ sometimes deposit pheromones even when they have inferior information than the agents encountered to prevent the system from falling into a local solution.

Here, we define the parameters as follows $\operatorname{mem}_{k}(t)$ : lost-competition counter

$\omega_{\text {mem }}$ : threshold for weak pheromone deposition

weak: pheromone value for weak pheromone deposition

In $A S_{\text {hierarchy, equations (2) and (3) are modified as }}$ follows:

$$
\begin{array}{ll}
\text { if ratio } \leq \varphi_{\text {ratio }}, & \ell_{x}(s, t) \geq \ell_{y}(s, t), \\
\text { then } \operatorname{mem}_{x} \leftarrow \operatorname{mem}_{x}+1, & C_{x} \leftarrow 0, \\
\text { if ratio } \leq \varphi_{\text {ratio }}, & \ell_{y}(s, t) \geq \ell_{x}(s, t), \\
\text { then } \text { mem }_{y} \leftarrow \operatorname{mem}_{y}+1, & C_{y} \leftarrow 0 .
\end{array}
$$

Equation (4) is also modified as follows:

$$
\tau_{i j}(t+1)=\rho \tau_{i j}(t)+ \begin{cases}\sum_{k} \frac{1}{L_{k}}, & \text { if }(i, j) \in T_{k}(t), C_{k}=1, \\ \sum_{k} \frac{\text { weak }}{L_{k}}, & \text { if }(i, j) \in T_{k}(t), C_{k}=0, \text { mem }_{k} \geq \omega_{\text {mem }}, \\ 0, & \text { otherwise, }\end{cases}
$$

where $\operatorname{mem}_{k}$ is reset to 0 at the beginning of each iteration $t$. Figure 1 shows the algorithm flowchart. The computational complexity of $A S_{\text {hierarchy }}$ can be almost similar with that of $A S_{\text {rank }}$ because agents in $A S_{\text {hierarchy }}$ need not be sorted according to tour lengths before pheromone updates; instead, the encounter between agents is considered.

\section{Results}

We conducted a tour exploration experiment on TSP benchmark datasets and compared the $A S_{\text {hierarchy }}$ simulation results with that of $A S_{\text {multi }}$ as we have already compared the performance of $A S_{\text {multi }}$ with $A S_{\text {rank }}$ [8]. We found that $A S_{\text {hierarchy }}$ yielded better solutions than $A S_{\text {rank }}$. We used four TSP datasets: Eil51.tsp $\left(N_{\text {city }}=51\right)$, Berlin52.tsp $\left(N_{\text {city }}=52\right)$, Lin 105.tsp $\left(N_{\text {city }}=105\right)$, and $\operatorname{Pr} 124 . t s p\left(N_{\text {city }}=124\right)$. Some of these datasets have often been used for benchmark tests $[14,15]$. The TSP datasets used here are classified as symmetric travelling salesman problem wherein the edge length between two cities in the opposite directions is the same. We focused on the TSP datasets adopting 2EUD. As shown in Table 1, the same values were adopted for the parameters common to $A S_{\text {multi }}$ and $A S_{\text {hierarchy }}$ [8]. $A S_{\text {hierarchy }}$ also has characteristic parameters $\omega_{\text {mem }}$ and weak; these parameter values were selected after a series of preliminary experiments comprising 10 attempts for each dataset. The parameters common to $A S_{\text {multi }}$ and $A S_{\text {hierarchy }}$ were set according to the previous study where we found that $A S_{\text {multi }}$ outperformed $A S_{\text {rank }}[8]$.

For each model, Table 2 shows the averaged best solutions obtained from 100 trials. In these benchmark datasets,
$A S_{\text {hierarchy }}$ outperformed $A S_{\text {multi }}$ for averaged best solutions. The $p$ value in Table 2 shows that there is significant difference between the averaged best solutions of both models in the $U$ test. In addition, the term "(optimal)" denotes the tour length of the optimal solution in each benchmark dataset. From the results in Table 2, it is seen that $A S_{\text {hierarchy, }}$ which allows agents who became a loser several times to update small quantities of pheromone, performs better than $A S_{\text {multi. }}$

Figure 2 shows the step interval of usual/weak pheromone depositions. We calculated the step interval of one agent using Eil51. According to Figure 2, the agent deposits weak pheromones more often than usual pheromones, satisfying equation (6). Such behaviours might inhibit rapid pheromone evaporation and result in the system getting away from local solutions.

Finally, we examined the parameter effect using Eil51. Here, we used the parameter value of $\omega_{\text {mem }}$ as 10 (instead of 5). Table 3 shows the results. Again, $A S_{\text {hierarchy }}$ performed better than $A S_{\text {multi }}$ even after the parameter replacement.

\section{Discussion}

In this paper, we investigated whether $A S_{\text {hierarchy }}$ outperforms $A S_{\text {multi. }} A S_{\text {multi }}$ is the basis for the proposed model. However, $A S_{\text {hierarchy allows agents who become a loser }}$ several times to update small quantities of pheromone. By contrast, agents in $A S_{\text {multi }}$ are never allowed to deposit any pheromones if they encounter other agents with better solutions. Weak pheromone depositions frequently occur in 


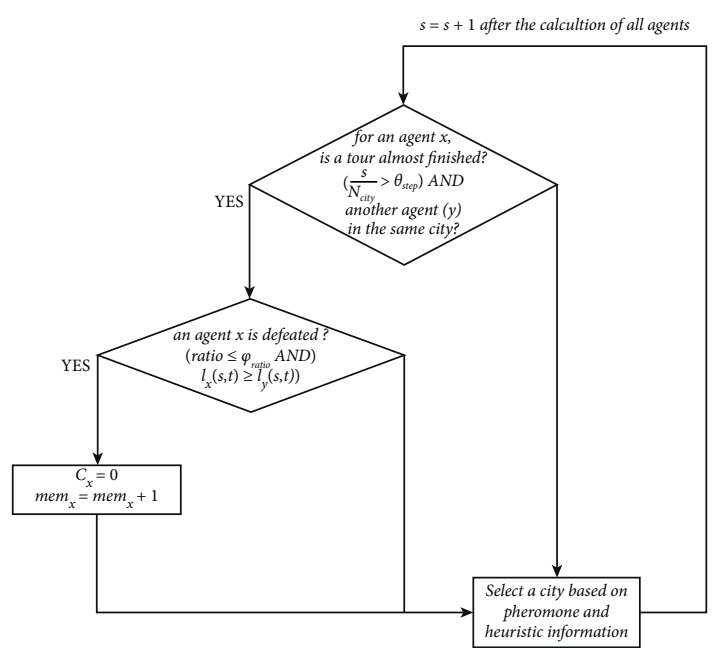

(a)

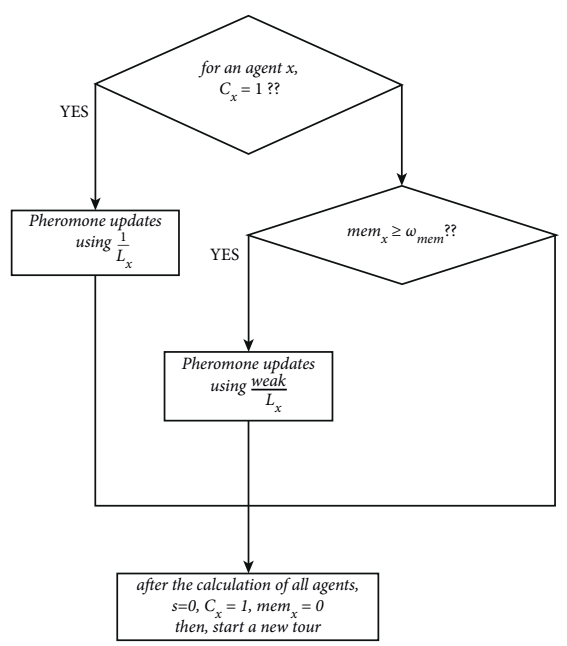

(b)

Figure 1: Flowcharts of $A S_{\text {hierarchy. }}$ (a) A tour iteration. (b) The pheromone update after each iteration. Note that ratio can be ratio $=\left|l_{x}(s, t)-l_{y}(s, t)\right| / l_{x}(s, t)+l_{y}(s, t)$, where $l_{x}(s, t)$ and $l_{y}(s, t)$ means accumulated tour length of agent $x$ and $y$ at step $s . L_{x}$ means tour length of solution the agent $x$ found.

TABle 1: Parameter settings.

\begin{tabular}{lcc}
\hline ACO model & $A S_{\text {hierarchy }}$ & $A S_{\text {multi }}$ \\
\hline$(\alpha, \beta)$ & $(1,1)$ & $(1,1)$ \\
P & 0.5 & 0.5 \\
Loop tour per 1 trial & 1000 & 1000 \\
Simulation trial & 100 & 100 \\
$\theta_{\text {step }}$ & 0.9 & 0.9 \\
$\varphi_{\text {ratio }}$ & 0.001 & 0.001 \\
$\omega_{\text {mem }}$ & 5 & - \\
Weak & 0.01 & - \\
\hline
\end{tabular}

TABLE 2: Mean best solutions.

\begin{tabular}{lccc}
\hline TSP & $A S_{\text {hierarchy }}$ & $A S_{\text {multi }}$ & $p$ value (optimal) \\
\hline Eil51 & 434.31 & 436.44 & $0.021(426)$ \\
Berlin52 & 7557.45 & 7589.5 & $0.047(7552)$ \\
Lin105 & 14630.34 & 14709.19 & $<1.0 e-06(14379)$ \\
Pr124 & 60334.46 & 60586.77 & $0.0067(59030)$ \\
\hline
\end{tabular}

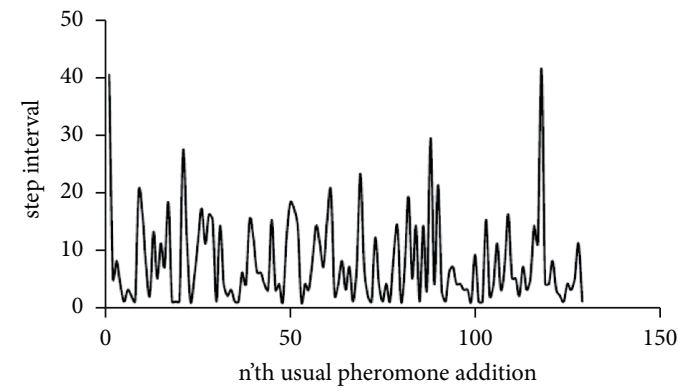

(a)

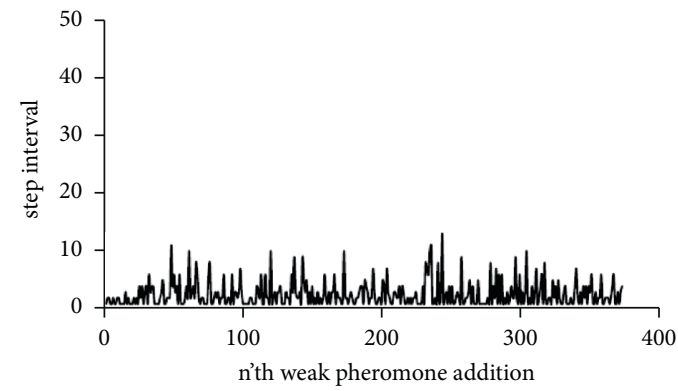

(b)

FIGURE 2: Step interval of pheromone addition (Eil51). (a) The step interval of the usual pheromone addition. (b) The step interval of weak pheromone addition. 
TABLE 3: Mean best solutions after parameter replacement.

\begin{tabular}{lccc}
\hline TSP & $A S_{\text {hierarchy }}$ & $A S_{\text {multi }}$ & $p$ value (optimal) \\
\hline Eil51 & 434.28 & 436.44 & $0.013(426)$ \\
\hline
\end{tabular}

$A S_{\text {hierarchy, }}$ which might improve the performance of $A S_{\text {hierarchy. }}$. The important point in the proposed model is that a group that has relatively better information than a specific agent does not always exhibit better information because agents are allowed to use only limited information via interaction with local other agents.

ACO does not guarantee convergence to the optimal solution $[16,17]$. Usual/weak pheromone depositions can serve as a key to achieve the balance between convergence and divergence to overcome this problem [18]. In this paper, we focused only on the proposed model's performance in terms of finding solutions; we did not consider other aspects such as the time required to find a correct solution or comparing our model with metaheuristics other than ACO. We would like to emphasise the importance of separation between third-party viewpoints and the first-person point of individual agents in multiagent systems. Researchers often introduce third-party viewpoints on metaheuristic systems derived from living systems that may not represent the original meaning of living/ natural systems to find better solutions. The strength of our findings is that individual agents decide to deposit pheromones based only on their experiences, which does not require seeing the system from a higher viewpoint. To do so, a system comprising many agents solves complex tasks flexibly without falling into a certain solution [19].

In future studies, we will investigate whether a stable solution can be obtained in other TSP datasets and find methods for parameter selection and for improving the algorithm's efficiency by considering the program execution steps.

\section{Data Availability}

The data used to support the findings of this study are available from the corresponding author upon request.

\section{Conflicts of Interest}

The authors declare no conflicts of interest.

\section{Authors' Contributions}

TS and IA conceived and designed the experiments; TS and KU developed algorithms; TS performed analysis and wrote the paper.

\section{Acknowledgments}

This study was partially supported by the Japan Society for the Promotion of Science (JSPS) (KAKENHI Grant no. 18K18343).

\section{References}

[1] M. Dorigo, M. Birattari, and T. Stützle, "Ant colony optimization," IEEE Computational Intelligence Magazine, vol. 1, pp. 28-39, 2016.

[2] M. Dorigo, Optimization, learning and natural algorithms (in Italian), Ph.D. Dissertation, Politecnico di Milano, Milan, Italy, 1992.

[3] M. Dorigo, "Ant colony system: a cooperative learning approach to the traveling salesman problem," IEEE Transactions on Evolutionary Computation, vol. 1, pp. 1-24, 1997.

[4] O. Cordo'n, I. F. de Viana, F. Herrera, and L. Moreno, A New ACO Model Integrating Evolutionary Computation Concepts: The Best-Worst Ant System, pp. 22-29, Universite' Libre de Bruxelles, Brussels, Belgium, 2000.

[5] C. Blum, A. Roli, and M. Dorigo, "HC-ACO: the hyper-cube framework for ant colony optimization," in Proceedings of MIC'2001-Metaheuristics International Conference, vol. 2, pp. 399-403, Porto, Portugal, May 2001.

[6] K. Socha, J. Knowles, and M. Sampels, "AMAX-MIN ant system for the university timetabling problem," in Ant Algorithms, M. Dorigo, G. Di Caro, and M. Sampels, Eds., Vol. 2463, Springer Verlag, Berlin, Germany, 2002.

[7] B. Bullnheimer, R. F. Hartl, and C. Strauss, "A new rank based version of the ant system: a computational study," Central European Journal of Operations Research, vol. 7, pp. 25-38, 1999.

[8] K. Uneme, T. Sakiyama, and I. Arizono, "Proposal of new ant system based on consistency and discrepancy of subjective ranking," in Proceedings of the International Multi-Conference of Engineers and Computer Scientists 2018, pp. 820-825, Hong Kong, China, March 2018.

[9] N. Miller, S. Garnier, A. T. Hartnett, and I. D. Couzin, "Both information and social cohesion determine collective decisions in animal groups," Proceedings of the National Academy of Sciences, vol. 110, no. 13, pp. 5263-5268, 2013.

[10] X. Cai, H. Zhao, S. Shang et al., "An improved quantuminspired cooperative co-evolution algorithm with mulistrategy and its application," Expert Systems with Applications, vol. 171, no. 9, Article ID 114629, 2021.

[11] W. Deng, S. Shang, X. Cai et al., "Quantum differential evolution with cooperative coevolution framework and hybrid mutation strategy for large scale optimization," KnowledgeBased Systems, vol. 224, Article ID 107080, 2021.

[12] W. Deng, J. Xu, H. Zhao, and Y. Song, "A novel gate resource allocation method using improved PSO-based QEA," IEEE Transactions on Intelligent Transportation Systems, vol. 99, pp. 1-9, 2020.

[13] W. Deng, J. Xu, X.-Z. Gao, and H. Zhao, “An enhanced MSIQDE algorithm with novel multiple strategies for global optimization problems," IEEE Transactions on Systems, Man, and Cybernetics: Systems, pp. 1-10, 2020.

[14] Y. Nakamichi and T. Arita, "Diversity control in ant colony optimization," Artificial Life and Robotics, vol. 7, pp. 198-204, 2014.

[15] T. Sakiyama, T. Hashimoto, and I. Arizono, "Ant colony optimization with function of autonomously switching rules 
of route selection," in Proceedings of the APIEMS 2017, pp. 1-5, CD-ROM, Jogjakarta, Indonesia, 2017.

[16] J. Walter and A. Gutjahr, "Graph-based ant system and its convergence," Future Generation Computer Systems, vol. 16, pp. $873-888,2000$.

[17] W. J. Gutjahr, "ACO algorithms with guaranteed convergence to the optimal solution," Information Processing Letters, vol. 82, no. 3, pp. 145-153, 2002.

[18] C. Detrain and J. Deneubourg, "Self-organized structures in a superorganism: do ants "behave" like molecules?" Physics of Life Reviews, vol. 3, no. 3, pp. 162-187, 2006.

[19] E. Bonabeau, M. Dorigo, and G. Theraulaz, Swarm Intelligence: From Natural to Artificial Systems, Oxford University Press, Oxford, UK, 1999. 